\title{
Localized pagetoid reticulosis
}

INSERM

\section{Source}

INSERM. (1999). Orphanet: an online rare disease and orphan drug data base. Localized pagetoid reticulosis. ORPHA:178517

Localized pagetoid reticulosis is a rare variant of mycosis fungoides (MF; see this term), a form of cutaneous T-cell lymphoma, and is characterized by the presence of localized patches or plaques with epidermal hyperplasia and intraepidermal proliferation of neoplastic T-cells, usually involving one extremity. 